\title{
一种基于协同效应的肼荧光探针及其在细胞成像中的应用
}

\author{
胥稳智* 李 雪韩孟楠周婷婷 \\ 杨瑜涛李 玮* \\ (河北大学化学与环境科学学院 药物化学与分子诊断教育部重点实验室 \\ 河北省化学生物学重点实验室 河北保定 071002)
}

\begin{abstract}
摘要 开发了一种基于协同效应的新型肼荧光探针. 通过在发色团上引入两种不同的识别位点, 双识别位点的协同效 应使得该探针对肼具有良好的专一性和灵敏度，检测限可达 $0.05 \sim 10.0 \mu \mathrm{mol} \cdot \mathrm{L}^{-1}$. 此外，该探针还可用于细胞 $(\mathrm{Bt}-474)$ 中肼的检测.
\end{abstract}

关键词 水合肼; 协同效应; 细胞成像; 荧光探针

\section{A Fluorescent Probe for Hydrazine Based on Synergistic Effect and Its Utilization in Cell Imaging}

\author{
Xu, Wenzhi* Li, Xue Han, Mengnan Zhou, Tingting Yang, Yutao Li, Wei* \\ (Key Laboratory of Medicinal Chemistry and Molecular Diagnosis of the Ministry of Education, Key Laboratory of Chemical \\ Biology of Hebei Province, College of Chemistry \& Environmental Science, Hebei University, Baoding, Hebei 071002)
}

\begin{abstract}
Hydrazine is an important raw material and catalyst in chemical industry. However, hydrazine is very harmful to human organs. A new type of probe was developed based on the synergistic effect. Two recognition sites were introduced into the probe to improve performance to hydrazine, which has good specificity to hydrazine. The limit of detection (LOD) for $\mathrm{N}_{2} \mathrm{H}_{4}$ was $0.05 \sim 10.0 \mu \mathrm{mol} \cdot \mathrm{L}^{-1}$. Moreover, the probe could detect $\mathrm{N}_{2} \mathrm{H}_{4}$ in BT-474 cells.

Keywords hydrazine; synergistic effect; cell imaging; fluorescent probe
\end{abstract}

肼的高还原性使其在医药、化工等领域得到了广泛 的应用 ${ }^{[1]}$. 肼有较高的燃烧热, 是一种有用的航空航天 推进剂 ${ }^{[2]}$. 然而, 肼对人体内脏有严重损伤作用，对人 体神经系统产生损伤 ${ }^{[3]}$. 动物实验证明, 肼还具有遗传 毒性. 美国环境保护署(EPA)规定饮用水中肼的含量应 低于 $0.312 \mu \mathrm{mol} \cdot \mathrm{L}^{-1[4]}$. 因此, 开发一种有效可靠的检测 肼的方法是十分必要的.

许多经典的方法已用于肼的检测，如气相色谱法、 电化学方法和紫外光谱法等. 然而, 这些方法存在操作 复杂及耗时等缺点 ${ }^{[5]}$. 与之相比, 荧光法具有灵敏度 高和操作简单等优点. 根据识别点, 我们可以将肼探针 分为乙酸酯类 ${ }^{[8 \sim 10]}$ 、醛类 ${ }^{[11 \sim 13]}$ 、氭基乙烯类 ${ }^{[14 \sim 16]}$ 、邻 苯二甲酰亚胺类 ${ }^{[17,18]} 、 4$-澳丁酸酯类 ${ }^{[19 \sim 21]}$ 、乙酰丙酸酯 类 ${ }^{[22-23]} 、 \beta$-二酮类 ${ }^{[24,25]} 、 \alpha, \beta$-不饱和羰基几类 ${ }^{[26-28]}$ 等 $^{[29-32]}$.
虽然文献已经报道多种反应型肼探针，这些探针仍有一 定的局限性，如选择性差(尤其是胺类化合物容易对肼 检测产生干扰)和对肼的灵敏度低等. 我们提出将双位 点协同效应引入到肼探针的设计中，在发色团上引入两 种不同的识别位点. 只有两种识别位点均与探针反应, 才能引起相应的苂光变化, 提高探针对肼的选择性. 另 外，利用两种不同的响应机制，可以产生两种效应的协 同，从而提高探针对肼的灵敏性. 近年来，我们 ${ }^{[33,34]}$ 报 道了两种基于协同效应的肼荧光探针，该探针对肼均具 有较高的选择性和敏感性. 作为这一策略的进一步研 究, 我们以 1,8 -䒬酰亚胺为苂光团, 设计合成了一种新 型肼探针(Scheme 1). 该探针的设计思想在于，同时采 用乙酸酯基和邻苯二甲酰亚胺作为识别位点, 近似 “双 锁原理”, 可以提高探针对肼的选择性; 对探针两种不

* Corresponding authors. E-mail: wzxu2008@sinano.ac.cn; liweihebeilab@163.com Received July 26, 2019; revised August 28, 2019; published online September 25, 2019.

Project supported by the National Natural Science Foundation of China (Nos. 21702043, 21807021).

国家自然科学基金(Nos. 21702043, 21807021)资助项目. 
同分子内电荷转移(ICT)过程进行调控, 两种效应的叠 加使得探针对肼的灵敏度得到了提高.

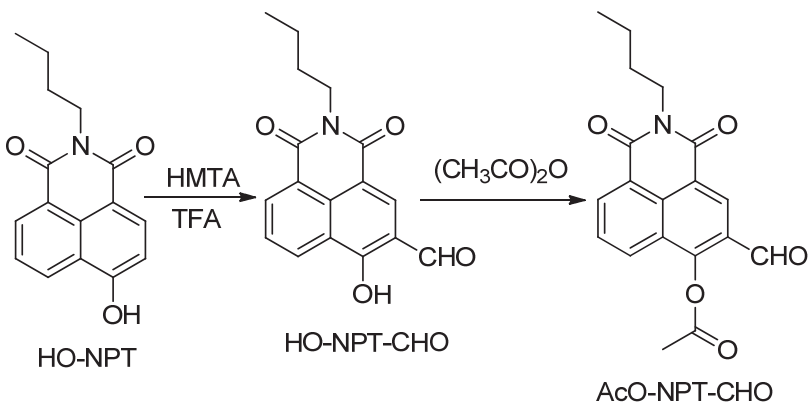

图式 1 探针的合成路线

Scheme 1 Synthetic procedure of the probe

\section{1 结果与讨论}

\section{1 紫外光谱和荧光光谱研究}

研究了在肼存在下探针的吸收光谱和发射光谱变 化. 实验结果显示, 游离探针在 $310 \mathrm{~nm}$ 处有最大吸收. 随着肼的加入, $310 \mathrm{~nm}$ 处的吸收峰逐渐减弱. 同时, 在 $454 \mathrm{~nm}$ 处出现新的吸收峰, 并在 $360 \mathrm{~nm}$ 处出现等吸收 点. 溶液的颜色由无色逐渐变为橙色, 显示该探针可用 于肼的比色检测.

通过苂光滴定实验对探针与肼的相互作用进行了 研究. 如图 1 所示, 在 $365 \mathrm{~nm}$ 激发波长下, 探针在 398 $\mathrm{nm}$ 处显示最大发射峰. 随着肼的添加, 探针在 $398 \mathrm{~nm}$ 处的苂光强度逐渐减弱, 而 $510 \mathrm{~nm}$ 处的苂光强度逐渐 增强. 当肼的浓度达到探针的 150 倍时, $510 \mathrm{~nm}$ 处的苂 光强度达到平衡. 随着肼的加入, 探针溶液的苂光颜色 逐渐由蓝色变为绿色. 在 $510 \mathrm{~nm}$ 处探针的苂光强度与 $\mathrm{N}_{2} \mathrm{H}_{4}$ 浓度 $\left(0.05 \sim 10.0 \mu \mathrm{mol} \cdot \mathrm{L}^{-1}\right)$ 呈线性关系 $\left(R^{2}=0.997\right)$. 根据 $\mathrm{LOD}=3 \sigma / k$, 计算出探针对肼的检测限为 0.014 $\mu \mathrm{mol} \cdot \mathrm{L}^{-1}$ ，低于美国环境保护署规定的 $0.312 \mu \mathrm{mol} \cdot \mathrm{L}^{-1}$.

\section{2 滴定体系 $\mathrm{pH}$ 節选}

为了获得更好的检测灵敏度, 分别对溶液的酸碱度 和反应时间进行了优化. 研究了不同 $\mathrm{pH}$ 值(3.0 12.0) 溶液中探针对肼的苂光响应. 实验结果显示, 在没有肼 的情况下, 在 $\mathrm{pH} \quad 3.0 \sim 7.0$ 的范围内探针没有明显的荧 光变化, 这说明探针本身在 $3.0 \sim 7.0$ 范围内是稳定的. 探针与肼作用后, 在 $\mathrm{pH} \quad 4.0 \sim 7.0$ 之间苂光强度变化较 大, 并且在 $\mathrm{pH}$ 值为 6 时荧光强度变化最大.

\section{3 选择性识别研究}

实际样品的复杂性要求该探针对肼的选择性必须 高于其它干扰物. 因此考察了多种可能的干扰物, 包括 各种阳离子 $\left(\mathrm{Mg}^{2+}, \mathrm{Hg}^{2+}, \mathrm{Ca}^{2+}, \mathrm{K}^{+}, \mathrm{Na}^{+}, \mathrm{NH}_{4}^{+}\right)$、阴离子 $\left(\mathrm{F}^{-}, \mathrm{Br}^{-}, \mathrm{NO}_{2}^{-}, \mathrm{OAc}^{-}\right)$、氨基酸 $(\mathrm{GSH}, \mathrm{Pro}, \mathrm{Hcy})$ 和胺类
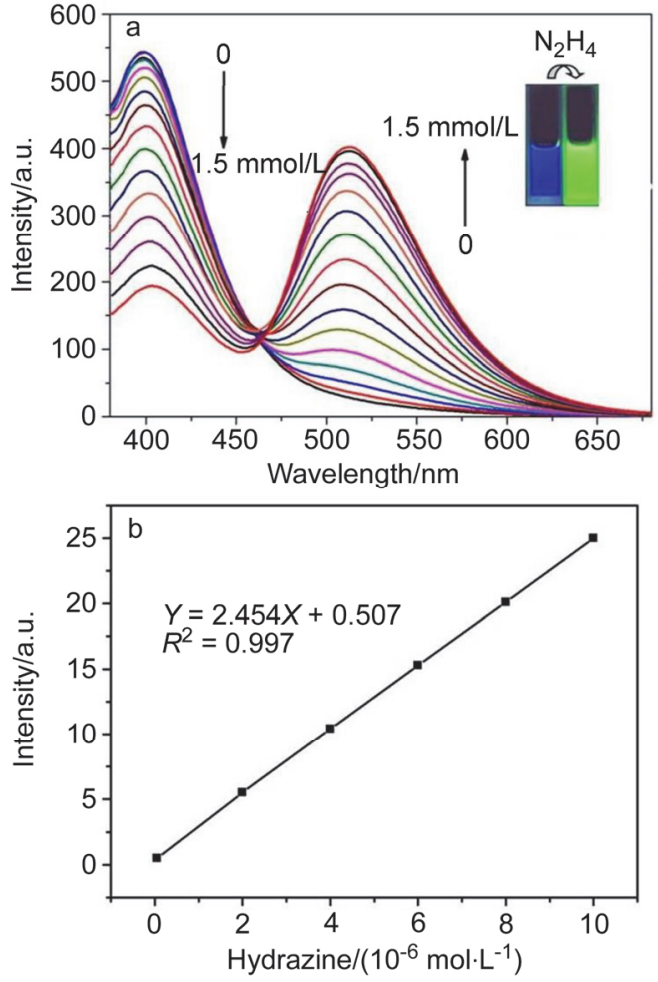

图 1 探针的荧光滴定曲线

Figure 1 Fluorescence titration curve of the probe

[semicarbazide hydrochloride (SEM), urea, isoniazid (Inh)]. 如图 2 所示, 除肼以外, 所有这些干扰物均未产 生显著的苂光变化. 随后，利用该探针对实际水样(自 来水)中的肼进行了检测. 分别在自来水和蒸馏水中加 入一定量的肼，调节 $\mathrm{pH}$ 至 6.0 , 测试苂光变化. 实验表 明，在一定浓度范围 $\left(2.5 \sim 7.5 \mu \mathrm{mol} \cdot \mathrm{L}^{-1}\right)$ 内，两种溶剂体 系的苂光强度基本一致. 在自来水和蒸馏水中肼的回收 率分别为 $97.20 \% \sim 99.60 \%$ 和 $99.20 \% \sim 102.60 \%$. 结果

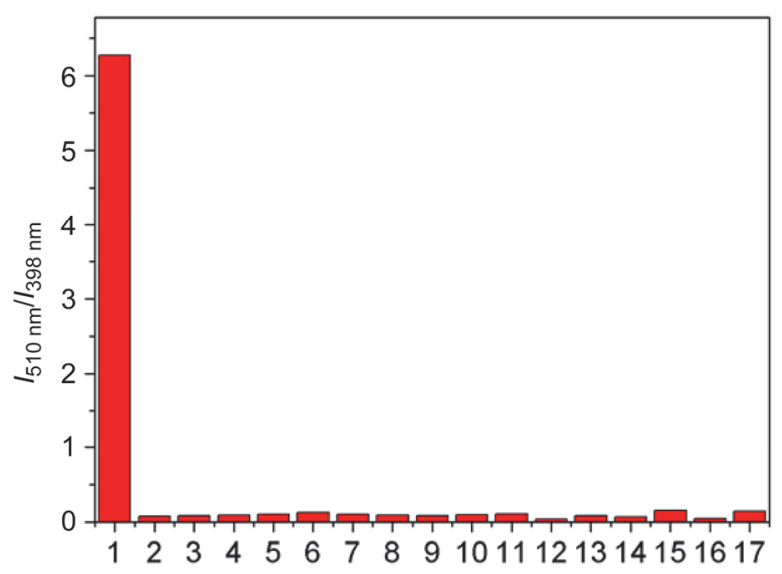

图 2 肼及各种干扰物对探针荧光强度的影响

Figure 2 Fluorescence response of hydrazine and various interfering substances to the probe 
表明, 该探针具有较强的抗干扰能力, 可用于实际水样 中肼的检测.

\section{4 识别机理研究}

为了阐明识别机理, 对识别产物进行了分离和表 征, 并与探针的谱图进行了对比. 对探针与产物的氢谱 进行比较发现, 探针在 $\delta 2.53$ 处的质子峰消失, 说明乙 酯基发生了肼解. 与此同时, 识别产物的芳香氢由 $\delta$ 8.62 向高场移至 $\delta 8.26$, 表明醛基转化为亚胺结构. 对 比探针与识别产物的红外光谱发现, 探针在 1767 和 $1704 \mathrm{~cm}^{-1}$ 处有红外吸收, 分别归属于酯羰基和醛羰基. 随着肼的加入, 1767 和 $1704 \mathrm{~cm}^{-1}$ 处的红外吸收消失, 而在 3400 和 $1694 \mathrm{~cm}^{-1}$ 处出现红外吸收, 这两个吸收峰 归属于腙. 实验结果证明, 酯基发生肼解, 同时醛基与 肼反应生成腙(Scheme 1). 利用高分辨质谱对识别产物 进行了确认(calcd for $\mathrm{C}_{17} \mathrm{H}_{18} \mathrm{~N}_{3} \mathrm{O}_{3}[\mathrm{M}+\mathrm{H}]^{+} 312.1348$, found 312.1341). 并且分别测定了识别产物的紫外光谱 和荧光光谱, 并与探针的紫外滴定和荧光滴定图相比 较, 发现二者基本吻合.

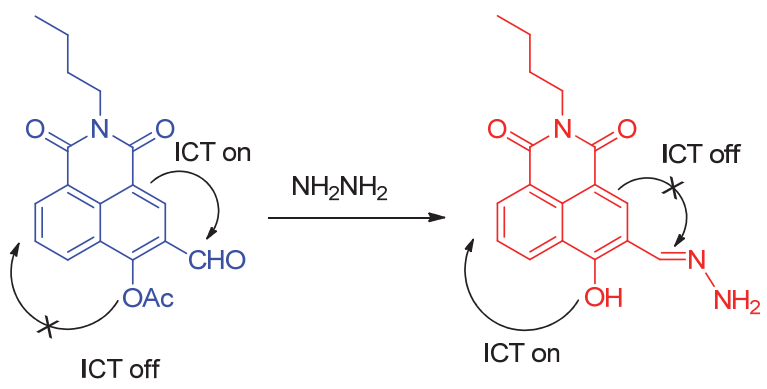

图式 2 可能的识别机理

Scheme 2 Proposed mechanisms for the detection of $\mathrm{N}_{2} \mathrm{H}_{4}$

\section{5 理论计算}

为了进一步将探针的结构变化与光学响应相关联, 利用高斯软件(Gaussian 09 program), 通过密度函数理 论 $(\mathrm{hf} / 3-21 \mathrm{~g}$ method)进行了计算. 结果表明, 在探针中 基态的电子云主要分布在䒺环部分, 而激发态的电子云 移至醛基部分，这表明在荥环与醛基之间存在着分子内 电荷转移(ICT). 与肼反应后, 基态电子主要分布在菜酚 部分, 在激发下电子会转移至萗环部分, 说明酚羟基与 萗环间同样存在着 ICT. 此外, 探针和产物最低空轨道 与最高已占轨道之间的能级差分别为 9.73 和 $9.48 \mathrm{eV}$, 该计算结果与紫外光谱的红移相一致.

\section{6 细胞成像研究}

鉴于该探针对肼具有良好的识别性能，将该探针应 用于细胞中肼的检测. 首先, 用噻唑蓝(MTT)法测定了 探针的细胞毒性. MTT 实验表明, 该探针对活细胞具有
较低的细胞毒性. 随后, 使用 BT-474 细胞来研究探针在 细胞成像中的实际应用. 当 BT- 474 细胞与探针 $(5 \mu \mathrm{mol} \cdot$ $\mathrm{L}^{-1}$ )共睬育 $24 \mathrm{~h}$ 后, 细胞呈蓝色荧光, 绿色通道内未见 荧光(图 3). 当 BT- 474 细胞与探针 $\left(5 \mu \mathrm{mol} \cdot \mathrm{L}^{-1}\right.$ )共捊育 24 $\mathrm{h}$ 后，再与不同浓度的 $\mathrm{N}_{2} \mathrm{H}_{4}\left(15,30\right.$ 和 $\left.45 \mu \mathrm{mol} \cdot \mathrm{L}^{-1}\right)$ 捊育 $1 \mathrm{~h}$, 绿色通道的荧光强度逐渐增强, 蓝色通道的苂光强 度逐渐减弱并消失. 细胞成像实验证明, 该探针可以用 于细胞中肼的检测. 虽然肼不是细胞的内源性物质，但 当人体服用某些药物如异烟肼后, 会在细胞新陈代谢过 程中释放出肼, 这是异烟肼产生毒副作用的重要原因. 通过该探针对细胞中肼的检测，可以个体化地限定服药 剂量, 防止药物中毒的发生, 同时该探针还有可能作为 异烟肼毒副作用的保护剂.

\section{2 结论}

基于双位点协同效应，我们设计合成出一种新型肼 荧光探针. 该探针对肼具有良好的选择性，各种可能的 干扰物如阳离子、阴离子、氨基酸和胺类均未其识别作 用产生影响. 该探针对肼的检测限为 $0.05 \sim 10.0 \mu \mathrm{mol}$ $\mathrm{L}^{-1}$, 低于 $\mathrm{EPA}$ 规定的 $0.312 \mu \mathrm{mol} \cdot \mathrm{L}^{-1}$. 细胞成像实验表 明，该探针可用于细胞中肼的检测.

\section{3 实验部分}

\section{1 仪器与试剂}

Bruker 核磁共振仪 (400 MHz); Apex Ultra 7.0T Bruker 质谱仪; Agilent G9800A 型荧光光谱仪(美国 Agilent 公司); UV-3600 型分光光度计(美国 Agilent 公 司); Thermo Nicolet iS10 红外光谱仪(美国 Thermo 公司); ZEISS LSM 880 激光共聚焦显微镜(德国 ZEISS 公司). 柱层析用硅胶(200 300 目), 实验室所用试剂均为市售 分析纯试剂.

\section{2 实验方法}

\subsection{1 化合物 HO-NPT-CHO 的合成}

将化合物 HO-NPT (1.000 g, $3.71 \mathrm{mmol}$ )溶解于 $5 \mathrm{~mL}$ 三氟乙酸中, 加入六次甲基四胺 $(1.041 \mathrm{~g}, 7.42 \mathrm{mmol})$, 加热至 $80{ }^{\circ} \mathrm{C}$ 回流反应 $12 \mathrm{~h}$. 冷却至室温, 将反应物倒 入冰水中, 有大量黄色固体析出. 抽滤, 并用冷的乙醇 洗涤, 干燥。粗产物经柱层析分离 $[V(\mathrm{DCM})$ : $V(\mathrm{MeOH})=100: 1$ ], 得 $750 \mathrm{mg}$ 黄色固体 HO-NPT-CHO, 产率 $60 \%$. m.p. $203.5 \sim 204.5{ }^{\circ} \mathrm{C} ;{ }^{1} \mathrm{H}$ NMR $\left(\mathrm{CDCl}_{3}, 400\right.$ MHz) $\delta: 13.21(\mathrm{~s}, 1 \mathrm{H}), 10.16(\mathrm{~s}, 1 \mathrm{H}), 8.75(\mathrm{dd}, J=8.0,4.0$ $\mathrm{Hz}, 3 \mathrm{H}), 7.84$ (t, $J=8.0 \mathrm{~Hz}, 1 \mathrm{H}), 4.21(\mathrm{t}, J=8.0 \mathrm{~Hz}, 2 \mathrm{H})$, $1.80 \sim 1.72(\mathrm{~m}, 2 \mathrm{H}), 1.55 \sim 1.45(\mathrm{~m}, 2 \mathrm{H}), 1.02(\mathrm{t}, J=8.0$ $\mathrm{Hz}, 3 \mathrm{H}) ;{ }^{13} \mathrm{C} \mathrm{NMR}\left(\mathrm{CDCl}_{3}, 400 \mathrm{MHz}\right) \delta: 196.42,165.65$, 

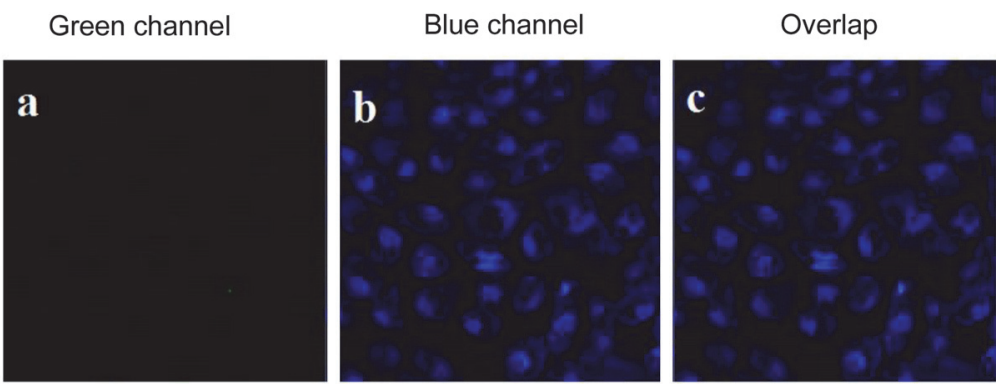

$0 \mu \mathrm{mol} \cdot \mathrm{L}^{-1}$

hydrazine
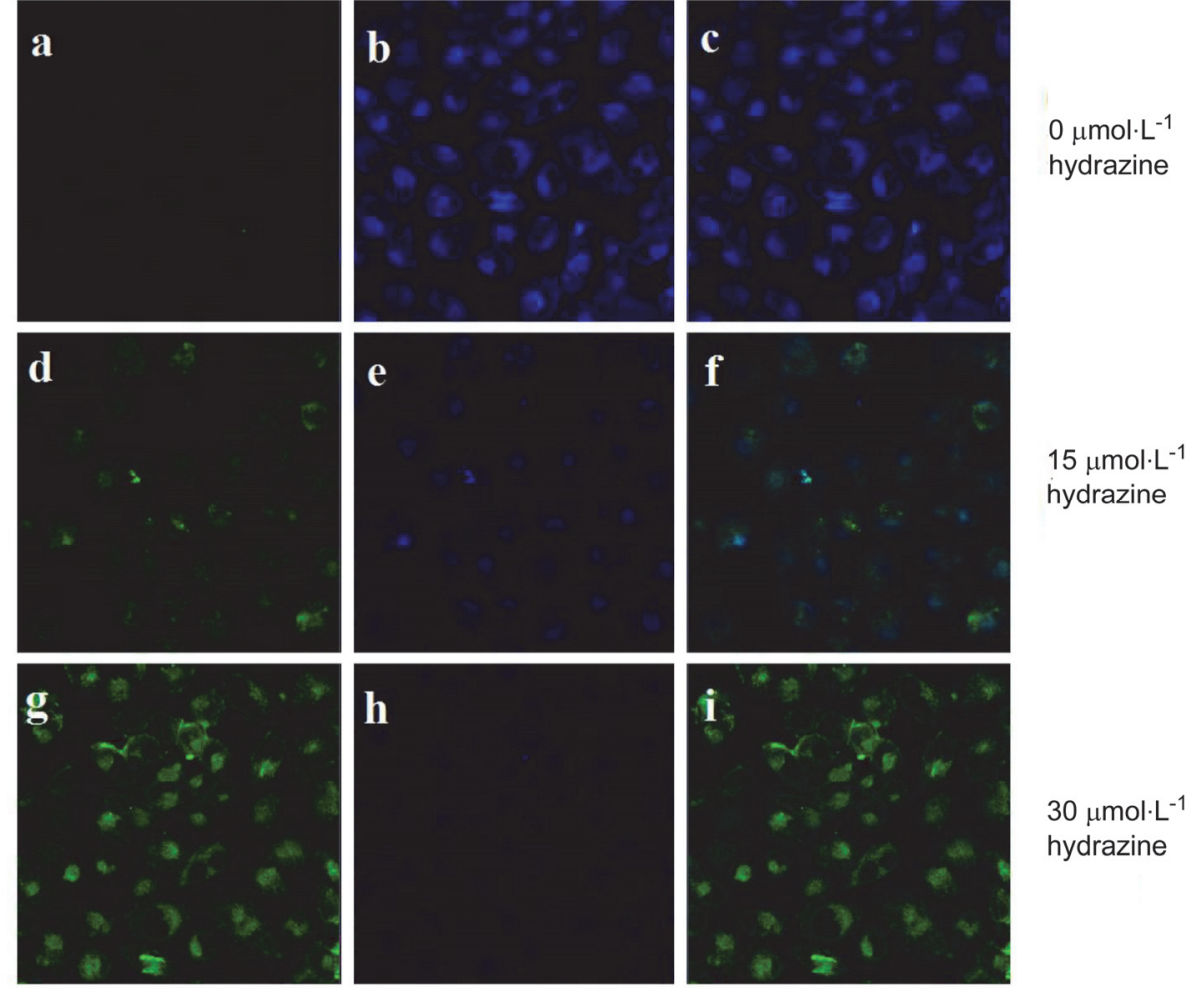

$15 \mu \mathrm{mol} \cdot \mathrm{L}^{-1}$

hydrazine
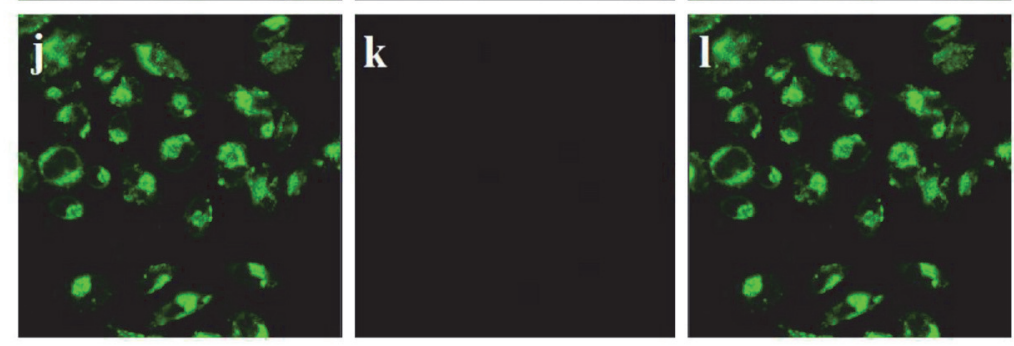

$30 \mu \mathrm{mol} \cdot \mathrm{L}^{-1}$

hydrazine

图 3 BT-474 细胞与探针和肼孵育的共聚焦图像

Figure 3 Confocal images of BT-474 cells incubated with the probe and further incubated with hydrazine

$163.64,162.98,134.84,134.07,131.74,130.29,127.01$, $122.84,122.81,115.14,114.87,40.29,30.18,20.35,13.83$; HRMS (ESI) cald for $\mathrm{C}_{17} \mathrm{H}_{14} \mathrm{NO}_{4}[\mathrm{M}-\mathrm{H}]^{-}$296.0928, found 296.0933 .

\subsection{2 探针 AcO-NPT-CHO 的合成}

将化合物 HO-NPT-CHO (500 mg, $1.68 \mathrm{mmol}$ )溶解 于 $2 \mathrm{~mL}$ 乙酸酐中, 加热至 $140{ }^{\circ} \mathrm{C}$ 回流反应 $12 \mathrm{~h}$. 冷却 至室温, 将反应液倒至冰水中, 加二氯甲烷萃取. 有机 相加无水硫酸钠干燥, 过滤, 蒸除溶剂, 柱层析分离 $[V(\mathrm{DCM}): V(\mathrm{MeOH})=100 ： 1]$, 得 $400 \mathrm{mg}$ 的黄色黄色 固体化合物 AcO-NPT-CHO, 产率 70\%. m.p. 193.8 $194.8{ }^{\circ} \mathrm{C} ;{ }^{1} \mathrm{H}$ NMR $\left(\mathrm{CDCl}_{3}, 400 \mathrm{MHz}\right) \delta: 8.62$ (s, 1H), $8.55(\mathrm{~d}, J=8.0 \mathrm{~Hz}, 1 \mathrm{H}), 8.40$ (d, $J=8.0 \mathrm{~Hz}, 1 \mathrm{H}), 8.01$ (s, 1H), 7.94 (t, $J=8.0 \mathrm{~Hz}, 1 \mathrm{H}), 4.04$ (t, $J=8.0 \mathrm{~Hz}, 2 \mathrm{H}), 2.57$ $(\mathrm{s}, 3 \mathrm{H}), 1.59 \sim 1.67(\mathrm{~m}, 2 \mathrm{H}), 1.34 \sim 1.40(\mathrm{~m}, 2 \mathrm{H}), 0.94(\mathrm{t}$, $J=8.0 \mathrm{~Hz}, 3 \mathrm{H}) ;{ }^{13} \mathrm{C} \mathrm{NMR}\left(\mathrm{CDCl}_{3}, 400 \mathrm{MHz}\right) \delta: 168.91$,
$168.45,163.70,163.15,149.34,132.30,130.04,129.46$, $128.37,127.91,126.59,125.66,123.06,121.50,84.24$, 40.35, 30.17, 20.72, 13.83; HRMS (ESI) calcd for $\mathrm{C}_{19} \mathrm{H}_{18^{-}}$ $\mathrm{NO}_{5}[\mathrm{M}+\mathrm{H}]^{+} 340.1179$, found 340.1176 .

\section{2 .3 光谱测试}

探针 AcO-NPT-CHO 用 $N, N$-二甲基甲酰胺(DMF)配 制溶液, 测试浓度为 $10 \mu \mathrm{mol} \cdot \mathrm{L}^{-1}$. 测试溶液均为 DMF-PBS 溶液 $\left(V: V=1: 1,10 \mathrm{mmol} \cdot \mathrm{L}^{-1} \mathrm{PBS}\right.$ 缓冲液, $\mathrm{pH}$ 8.0), 选择性测试所用各种干扰离子均为钠盐或钾 盐, 测试浓度均为 $15.0 \mathrm{mmol} \cdot \mathrm{L}^{-1}$. 紫外光谱和苂光光谱 均在室温下测试, 样品池为 $1 \mathrm{~cm} \times 1 \mathrm{~cm} \times 4 \mathrm{~cm}$ 石英比 色典，激发波长为 $365 \mathrm{~nm}$, 激发和发射狭缝宽度均为 5 $\mathrm{nm}$.

\subsection{4 细胞实验}

Bt-474 细胞接种于成像专用的细胞培养血, 密度为 $2 \times 10^{5}$ 个/孔. 探针组加入探针 AcO-NPT-CHO (5 
$\left.\mu \mathrm{mol} \cdot \mathrm{L}^{-1}\right)$ 孵育 $24 \mathrm{~h}$. 外源肼 $\left(\mathrm{N}_{2} \mathrm{H}_{4}\right)$ 成像组分别加入 15 , $30,45 \mu \mathrm{mol} \cdot \mathrm{L}^{-1}$ 继续孵育 $1 \mathrm{~h}$. 共聚焦荧光成像激发波 长为 $365 \mathrm{~nm}$, 收集范围 450 500 nm.

辅助材料 (Supporting Information) 所合成化合物 HO-NPT-CHO, AcO-NPT-CHO 及识别产物的核磁、质 谱、红外谱图. 这些材料可以免费从本刊网站(http:// sioc-journal.cn/)上下载.

\section{References}

[1] Ragnarsson, U. Chem. Soc. Rev. 2001, 30, 205.

[2] Mo, J. W.; Ogorevc, B.; Zhang, X.; Pihlar, B. Electroanalysis 2000, $12,48$.

[3] Reilly, C. A.; Aust, S. D. Chem. Res. Toxicol. 1997, 10, 328.

[4] U. S. Environmental Protection Agency (EPA) Integrated Risk Information System (IRIS) on Hydrazine/Hydrazine Sulfate, National Center for Environmental Assessment, Office of Research and Development, Washington, DC, 1999.

[5] McAuley, C. B.; Banks, C. E.; Simm, A. O.; Jones, T. G.; Compton, R. G. Analyst 2006, 131, 106.

[6] Sun, M.; Bai, L.; Liu, D. Q. J. Pharm. Biomed. Anal. 2009, 49, 529.

[7] Kawasaki, T.; Maeda, M.; Tsuji, A. J. Chromatogr., Biomed. Appl. 1979, 163, 143

[8] Qu, P.; Ma, X.; Chen, W. S.; Zhu, D. D.; Bai, H. F.; Wei, X. H.; Shu, C.; Xu, M. T. Spectrochim. Acta, A 2019, $210,381$.

[9] Liu, C.; Liu, K. Y.; Tian, M. G.; Lin, W. Y. Spectrochim. Acta, A 2019, 212, 42 .

[10] Song, Y. Q.; Chen, G.; Han, X. Y.; You, J. M.; Yu, F. B. Sens. Actuators, $B$ 2019, 286, 69.

[11] Purohit, D.; Sharma, C. P.; Raghuvanshi, A.; Jain, A.; Rawat, K. S.; Gupta, N. M.; Singh, J.; Sachdev, M.; Goel, A. Chem.-Eur. J. 2019, 25,4660 .

[12] Jung, Y.; Ju, I. G.; Choe, Y. H.; Kim, Y.; Park, S.; Hyun, Y.; Oh, M. S.; Kim, D. ACS Sens. 2019, 4, 441.

[13] Meher, N.; Panda, S.; Kumar, S.; Iyer, P. K. Chem. Sci. 2018, 9, 3978.

[14] Liu, Y.; Ren, D. D.; Zhang, J. J.; Xi, H. Li.; Yang, F. Dyes Pigm. 2019, 162, 112 .

[15] Paul, S.; Nandi, R.; Ghoshal, K.; Bhattacharyya, M.; Maiti, D. K. New J. Chem. 2019, 43, 3303.

[16] Wu, J. J.; Pan, J.; Ye, Z.; Zeng, L. T.; Su, D. D. Sens. Actuators, B
2018, 274, 274.

[17] Wang, W. D.; Hu, Y.; Li, Q.; Hu, S. L. Inorg. Chim. Acta 2018, 477, 206.

[18] Maji, R.; Mahapatra, A. K.; Maiti, K.; Mondal, S.; Ali, S. S.; Sahoo, P.; Mandal, S. H.; Uddin, M. R.; Goswami, S.; Quah, C. K.; Fun, H. K. RSC Adv. 2016, 6, 70855 .

[19] Wu, Q. Q.; Zheng, J. L.; Zhang, W. C.; Wang, J. B.; Liang, W. L.; Stadler, F. J. Talanta 2019, 195, 857.

[20] Lu, Z. L.; Shi, X. M.; Ma, Y. F.; Fan, W. L.; Lu, Y. N.; Wang, Z.; Fan, C. H. Sens. Actuators, B 2018, 258, 42.

[21] Lv, H. S.; Sun, H. Y.; Wang, S. J.; Kong, F. G. Spectrochim. Acta, A 2018, 196, 160.

[22] Tiensomjitr, K.; Noorat, R.; Chomngam, S.; Wechakorn, K.; Prabpai, S.; Kanjanasirirat, P.; Pewkliang, Y.; Borwornpinyo, S.; Kongsaeree, P. Spectrochim. Acta, A 2018, 195, 136.

[23] Ju, Z. Y.; Li, D. P.; Zhang, D.; Li, D. D.; Wu, C. Z.; Xu, Z. H. J. Fluoresc. 2017, 27, 1.

[24] Wu, W. N.; Wu, H.; Wang, Y.; Mao, X. J.; Zhao, X. L.; Qing, Z.; Xu, Q.; Fan, Y. C.; Xu, Z. H. Spectrochim. Acta, A 2018, 188, 80.

[25] Roy, B.; Halder, S.; Guha, A.; Bandyopadhyay, S. Anal. Chem. 2017, 89, 10625

[26] Luo, Z. J.; Liu, B.; Qin, T. Y.; Zhu, K. N.; Zhao, C.; Pan, C. J.; Wang, L. Sens. Actuators, B 2018, 263, 229.

[27] Xu, H.; Gu, B.; Li, Y. Q.; Huang, Z.; Su, W.; Duan, X. L.; Yin, P.; Li, H. T.; Yao, S. Z. Talanta 2018, 180, 199.

[28] Liu, L.; Le, Y.; Teng, M. G.; Zhou, Z. X.; Zhang, D.; Zhao, C. S.; Cao, J. X. Dyes Pigm. 2018, 151, 1.

[29] Yang, Z. Q.; Liu, X. K.; Jiang, L. N.; Wang, M. Chin. J. Org. Chem. 2019, 39, 1483 (in Chinese). (杨滋琦, 刘兴坤, 姜鲁南, 王美, 有机化学, 2019, 39, 1483.)

[30] Ju, Z. Y.; Shu, P. H.; Xie, Z. Y.; Jiang, Y. Q.; Tao, W. W.; Xu, Z. H. Chin. J. Org. Chem. 2019, 39, 697 (in Chinese)

(鞠志宇，舒朋华，谢智宇，蒋雨晴，陶伟杰，许志红，有机化学， 2019, 39, 697.)

[31] Zhang, Y.; Liu, J. F.; Yi, R. H.; Ai, S. F.; Jia, W. Z. Chin. J. Anal. Chem. 2018, 46, 511 (in Chinese). (张勇，刘劲风，易润豪，艾思凡，贾文志，分析化学，2018，46, 511.)

[32] Wang, R. X.; Lai, X. J.; Qiu, G. Y. S.; Liu, J. B. Chin. J. Org. Chem. 2019, 39, 952 (in Chinese). (王瑞祥，赖晓静，邱观音生，刘晋彪，有机化学, 2019, 39, 952.)

[33] Xu, W. Z.; Liu, W. Y.; Zhou, T. T.; Yang, Y. T.; Li, W. Spectrochim. Acta, A 2018, 193, 324.

[34] Xu, W. Z.; Liu, W. Y.; Zhou, T. T.; Yang, Y. T.; Li, W. J. Photochem. Photobiol. A 2018, 356, 610. 